\title{
Towards an Autonomous Unwrapping System for Intralogistics
}

\author{
Chiara Gabellieri ${ }^{1}$, Alessandro Palleschi ${ }^{1}$, Anna Mannucci ${ }^{1}$, Michele Pierallini ${ }^{1}$, Elisa Stefanini ${ }^{1}$, \\ Manuel G. Catalano ${ }^{2}$, Danilo Caporale ${ }^{1}$, Alessandro Settimi ${ }^{1}$, Todor Stoyanov ${ }^{3}$, \\ Martin Magnusson $^{3}$, Manolo Garabini ${ }^{1}$, and Lucia Pallottino ${ }^{1}$
}

\begin{abstract}
Warehouse logistics is a rapidly growing market for robots. However, one key procedure that has not received much attention is the unwrapping of pallets to prepare them for objects picking. In fact, to prevent the goods from falling and to protect them, pallets are normally wrapped in plastic when they enter the warehouse. Currently, unwrapping is mainly performed by human operators, due to the complexity of its planning and control phases. Autonomous solutions exist, but usually they are designed for specific situations, require a large footprint and are characterized by low flexibility. In this work, we propose a novel integrated robotic solution for autonomous plastic film removal relying on an impedance-controlled robot. The main contribution is twofold: on one side, a strategy to plan Cartesian impedance and trajectory to execute the cut without damaging the goods is discussed; on the other side, we present a cutting device that we designed for this purpose. The proposed solution presents the characteristics of high versatility and the need for a reduced footprint, due to the adopted technologies and the integration with a mobile base. Experimental results are shown to validate the proposed approach.
\end{abstract}

Index Terms-Logistics, Compliance and Impedance Control, Industrial Robots, Automatic Unwrapping

\section{INTRODUCTION}

$\mathbf{R}$ OBOTICS-LOGISTICS, the field of logistic processes automated by robotic solutions, is divided in four main phases, as discusses in [1] and [2]: Pallet Unloading, Depalletizing, Palletizing, and Pallet Loading. Intralogistics refers to the management and optimization of the internal flow of goods, that includes De-palletizing and Palletizing phases within a company premises [2], [3], [4].

According to a 2014 study from the Freedonia Group [5], the number of pallets in use globally were projected to grow

Manuscript received: February, 24, 2019; Revised April, 30, 2019; Accepted April, 30, 2019.

This paper was recommended for publication by Editor J. Li upon evaluation of the Associate Editor and Reviewers' comments. This work has received funding from the European Union's Horizon 2020 research and innovation program under agreement no. 732737 (ILIAD) and by the Italian Ministry of Education, and Research (MIUR) in the framework of the CrossLab project (Departments of Excellence). The content of this publication is the sole responsibility of the authors. The European Commission or its services cannot be held responsible for any use that may be made of the information it contains. The authors thank Bastien Veyssiere for its work in the perception part.

1 Centro di Ricerca E. Piaggio e Departimento di Ingnegneria dell'Informazione, Universita' di Pisa, Pisa, Italia lucia.pallottino@unipi.it

${ }^{2}$ Istituto Italiano di Tecnologia, via Morego, 30, 16163 Genova, Italy

${ }^{3}$ Centre for Applied Autonomous Sensor Systems (AASS), Orebro University, Sweden

Digital Object Identifier (DOI): see top of this page.

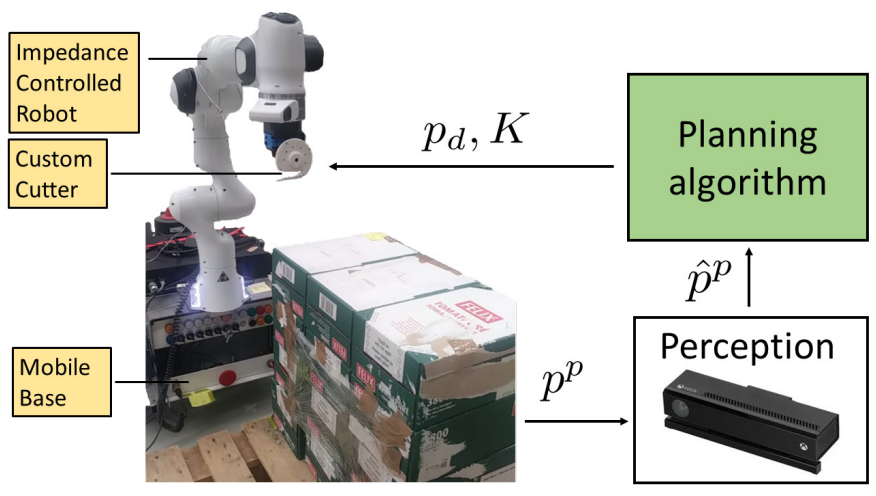

Fig. 1. Architecture of the envisioned system: the perception system acquires information of the pallet $\left(p^{p}\right)$ and provides an estimate to the planning algorithm $\left(\hat{p}^{p}\right)$, which computes reference trajectory and Cartesian impedance $\left(p_{d}, K\right)$ for the impedance-controlled robot mounted on a mobile base and equipped with the custom cutting end-effector.

up to 9.9 billion units through 2017, with an annual expected increase of 4.6 percent. More recent studies ([6], [7]) confirm the increasing trend in the global sales of pallets, and forecast a CAGR ${ }^{1}$ up to $5.4 \%$ until 2025.

Given such numbers, it is easy to understand how, today, intralogistic services are required to respond quickly to evolving market needs. To meet these requirements, logistics stakeholders demand a new generation of intralogistic systems that are flexible, efficient, and safe in environments shared with humans.

This work focuses on the unwrapping, the first task of the intralogistic process, a preliminary and necessary removal of the plastic film enclosing the pallettized goods in order to make them accessible for manipulation.

Due to the perception and control challenges of this task (for a detailed discussion see Sec. III), unwrapping is particularly far from complete automation in the current industrial scenario. The very few examples of autonomous solutions (described in Sec. II) suffer from two major limitations: they are bulky machines limiting the efficiency of the warehouse, and are only suitable for properly designed pallets.

In this paper, we propose a novel solution to automatic unwrapping (see Fig.1), composed by a perception system, an impedance-controlled robot mounted on a mobile base,

\footnotetext{
${ }^{1}$ Compound annual growth rate (CAGR) is a business and investing specific term for the geometric progression ratio that provides a constant rate of return over the time period.
} 


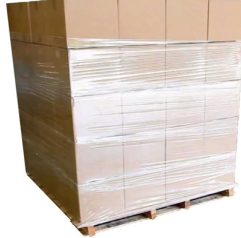

(a) Lateral wrapping (Case1)

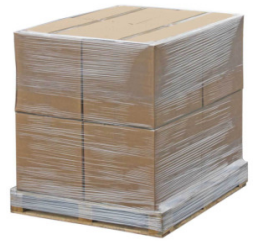

(b) \{Incomplete top wrapping (Case2)

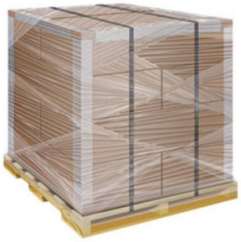

(c) Complete wrapping (Case 3)
Fig. 2. Examples of different wrapping cases: the film covers only the lateral surface of the pallet (a), the film also covers the top surface partially (b), the film covers the lateral and the top surface entirely (c).

a custom cutter end-effector (e.e.), and a planning strategy. Compared to the state of the art solutions, our proposal has a small footprint and it is mobile, hence can be relocated to maximize the warehouse efficiency. Furthermore, even if at the present state we handle rectangular pallets only (which however covers a large range of practical situations), the system is intended to cope with pallets having a wide variety of stacking configurations (cylinders, and arbitrarily shaped pallets in general). The main contribution is twofold: on one side a strategy to plan Cartesian impedance and trajectory to effectively execute the cut of the wrapping film without damaging the goods under uncertain conditions (presented in Sec. IV and V); on the other side a cutting device is presented in Sec. VI, which has been designed for this purpose after extensive experimental tests of existing tools featuring different working principles (results are reported in the Appendix). Among the three ways in which a pallet may be wrapped (see Fig. 2), the complete wrapping is equivalent to the incomplete top wrapping, provided that a hole is created in the film covering the top face. The proposed solution has been tested in the lateral wrapping, and in the incomplete top wrapping cases, showing a success rate of $100 \%$ and $78 \%$, respectively. The results of the experiments are presented and discussed in Sec. VII. Finally, the conclusions are drawn in Sec. VIII, with an overview of planned future work.

\section{StATE OF ART}

Integrated solutions for automatic depalletizers, i.e. machines that accomplish the unwrapping phase and the unpacking of the palletized object, require the presence of a human operator for the unwrapping phase $^{2}$. However, automatic unwrapping solutions are available in the industry. An example is the $\mathrm{MSK}^{3}$ fully automatic unwrapping machine, which is composed of a portal frame under which the pallet to be unwrapped is placed. A large metal structure cuts the wrapping sheet. After that, a cylinder that is connected to the portal rotates around the pallet and winds the sheet. Finally, the sheet is suctioned under the machine to free the cylinder for the next operation. Another example is the BWContainer Systems ${ }^{4}$ automatic unwrapping machine, composed of two

\footnotetext{
${ }^{2}$ See e.g. http://www.maspack.com/en/product/tato-r-400-bpm/.

${ }^{3}$ https://www.mskcovertech.com/solutions/unwrapping-

systems/unwrapping-machine-beverages/

${ }^{4}$ https://www.bwintegratedsystems.com/machine/materialhandling/destrapping/robotic-unwrapper
}

large anthropomorphic manipulators. One is responsible for suctioning the wrapping sheet with vacuum grippers while the other performs the cut. After the cutting phase is completed, one arm picks a limb of the wrapping film and places it on a suction machine that frees the pallet. Further examples apply to specific objects. Among these, the automatic unwrappers by Autorema ${ }^{5}$ and the CSW-Multifeeder ${ }^{6}$ series are designed for packets of end-cans.

These machines have two main drawbacks: they are bulky, thus reducing the surface of the warehouse available to store products, and they work with a structured and specific design of the pallet hence limit the flexibility of the system.

\section{Challenges tOWARDS Flexible UnWRAPPING}

The unwrapping task can be divided into a sequence of subtasks, namely pallet detection, film detection, film cutting, and film removal, as represented in Fig. 3

The pallet detection phase has been already addressed in several previous works. In [8] the authors present ROBOLIFT: an autonomous fork lift carrier able to perform pallets recognition and to estimate the pallet pose. In [9] an autonomous outdoor robotic forklift is able to safely engage unknown pallets, while in [10] the focus is put on the perception system for autonomous pallet detection to be applied in intralogistic operations. In [10] the reader can find references to pallet detection methods based on different working principles, such as laser scanners, vision-based, or relying on sensor fusion.

The goal of the film detection phase is to estimate which portion of the surface of the pallet is covered by the wrapping material. A pallet can be wrapped in three different possible ways (see Fig.2). In the case named lateral wrapping, only the lateral surface of the pallet is partially covered by the film; in the incomplete top wrapping case the lateral surface is completely covered, while the top surface is partially covered; in the complete wrapping case also the top surface of the pallet is completely covered. The challenge in this phase is to reliably distinguish the plastic film, independently from light conditions, object materials, object colors, film layers. To the best of our knowledge, plastic film detection is not solved except for cases with fixed materials and light setups. State-of-the-art methods for general surface reflectance characterisation [11], [12] require custom-built light and detector setups. Existing industrial sensors, such as the SICK OPR20G glare sensor, only work in very close proximity to the material. Neither of these approaches allow for easy deployment on a mobile robot in a warehouse setting. In ongoing work, we are investigating how to detect plastic also in more general and uncontrolled, warehouse-like, scenarios. For the present work we assume that there is a perception module that can detect, with some variance, which points on a pallet are wrapped and which are not.

In the film cutting phase the system executes the cutting task while preserving the integrity of the items. The autonomous and efficient execution of this phase, which represents an open problem, is the focus of this work. In this phase the pallet size

\footnotetext{
${ }^{5}$ https://autorema.com/en/can-making-industry/

${ }^{6}$ https://cswbv.com/multifeeder-4/
} 


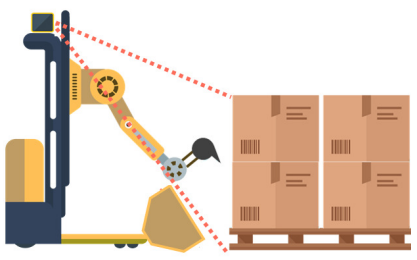

(a) pallet detection

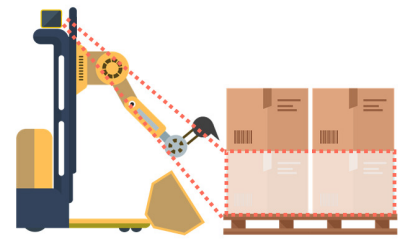

(b) film detection

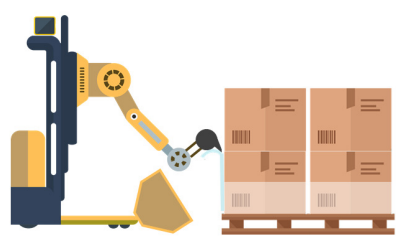

(c) film cutting

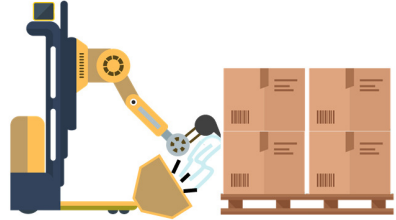

(d) film removal

Fig. 3. Four main phases of the unwrapping task. the pallet position and size,(a), and the plastic film distribution, (b), are acquired by a perception system, then the plastic film is cut, (c) and it is removed,(d).

and pose, together with the distribution of the wrapping film, should be used to plan and control the system motion. Due to the variety of items (shape, size, colors, surfaces, etc.) that can be stacked in the pallets, the information coming from the perception system will be affected by a certain degree of uncertainty. This poses two main challenges: ensuring that, when the robot comes in contact with the objects, it does not cause them any damage; after establishing the engagement between the plastic film and the robot end-effector, efficiently accomplishing the task, possibly keeping the engagement until the end of the task. Given the tight wrapping, to establish (and possibly not loosing) the engagement, it is desirable that a certain level of interaction between the end-effector and the palletized items is allowed. However, to preserve the integrity of the items, it is mandatory that the robot does not exert an interaction force on the goods larger than a given threshold, but also that the design of the cutter prevents the cutting element to come in contact with the items.

The film removal phase can be addressed by exploiting one of the solutions available in the state of the art of automatic unwrapping: it might be collected and suctioned by a vacuum machine. However, we will investigate also the possibility of solving this sub-task exploiting a manipulation system.

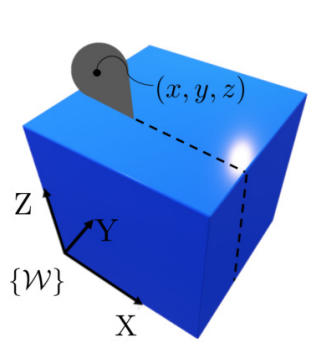

(a)

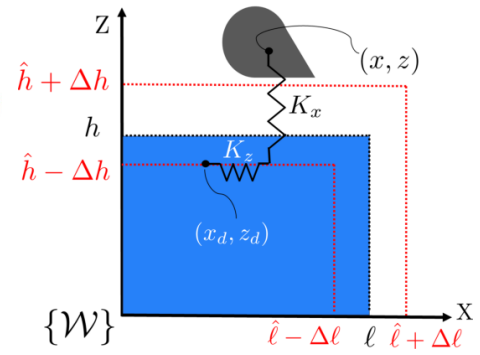

(b)
Fig. 4. The cutter (grey surface) is represented, with an approximation of the pallet as a box (blue surface) and the world-fixed frame, $\{\mathcal{W}\}$. On the left: a 3-D representation, the dashed line indicates a possible trajectory of the cutter. On the right: a view in the XZ plane. Virtual impedances $K_{x}$ and $K_{z}$ connect the cutter, point $(x, z)$, with the point on the planned trajectory, $\left(x_{d}, z_{d}\right)$. The dimensions of the pallet lie inside uncertainty regions, whose bounds are the red dotted lines.

\section{IMPEDANCE PLANNING: PROBLEM STATEMENT}

Based on the analysis of the task challenges (reported in Sec. III), the goal of the planning system is that the endeffector successfully engages the plastic film and complete the cutting task without exerting an excessive force on the palletized items under the uncertainty affecting the knowledge of pallet position and size, and of the plastic film distribution.

To handle the interaction forces between the cutter and the objects a pure position control, making the robot stiff during the physical interaction, is not the most suitable solution, and would result in a very slow motion required to preserve the integrity of the goods. To overcome this issue, we choose to adopt a Cartesian impedance controlled robot. In the literature several impedance control approaches have been presented ([13], [14]), here we assume that the robot is controlled by a Cartesian impedance law with reshaping of the inertia matrix and contact force compensation, as in Sec. 9 of [15]. Considering a point-mass model of the cutter in contact with the environment, the operational space dynamics of such a system can be written as:

$$
\begin{aligned}
& M_{d}\left(\ddot{p}-\ddot{p}_{d}\right)+D\left(\dot{p}-\dot{p}_{d}\right)+K\left(p-p_{d}\right)=F_{e}+L(p)^{T} F_{\perp}, \\
& L(p) \dot{p}=0
\end{aligned}
$$

where $p(t)=[x(t) y(t) z(t)]^{T} \in \mathbb{R}^{3}$ is the configuration vector, $\dot{p}$ its first time-derivative, $\ddot{p}$ its second time-derivative, the subscript $(\cdot)_{d}$ denotes the corresponding desired quantities. $M_{d} \in \mathbb{R}^{3 \times 3}, D \in \mathbb{R}^{3 \times 3}$, and $K=\operatorname{diag}\left(K_{x}, K_{y}, K_{z}\right) \in \mathbb{R}^{3 \times 3}$ are the desired diagonal inertia, damping, and stiffness matrices, respectively; $L \in \mathbb{R}^{1 \times 3}$ identifies the direction orthogonal to the surface of the pallet at the contact point; $F_{\perp} \in \mathbb{R}$, with $F_{\perp} \geq 0$, is the constraint reaction force in that direction; $F_{e} \in \mathbb{R}^{3}$ is the external force vector.

Assuming a cuboid approximation of the pallet, a scheme of the cutting task is reported in Fig. 4 where $h$ represents the actual pallet height, and $\hat{h}$ represents the height estimate provided by the perception system subject to the uncertainty $\Delta h ; \ell$ is the actual pallet length, and $\hat{\ell}$ is the length estimate subject to the uncertainty $\Delta \ell$.

The problem is to plan both the trajectory and the impedance. Note that assuming a desired critically damped behavior of the robot, once $K$ has been set, $D$ and $M_{d}$ are computed consequently. Hence the problem is reduced to the planning of $K$ and $p_{d}$ under a set of constraints given by the bounds on the stiffness and on the contact forces intensity.

\section{IMPEDANCE PLANNING: SOLUTION}

We now propose a planning strategy in order to autonomously complete the cutting phase of the unwrapping task. We do not describe the complete wrapping case because 

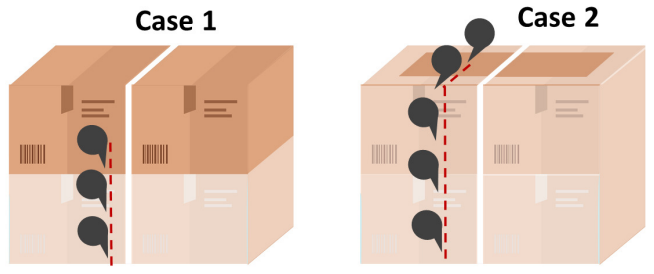

Fig. 5. Two configurations of the film. Case 1 corresponds to the lateral wrapping case, and Case 2 to the incomplete wrapping case.

the approach would be the same as for the incomplete top wrapping, once a hole is produced in the film on the top face of the pallet, from which initiating the cut. Depending on the wrapping case the simplest path that the end-effector may follow is (see Fig. 5):

- Lateral Wrapping: the planned path is composed of a line on the lateral surface.

- Incomplete Top Wrapping: the planned path is composed of two lines, one on the top surface, and one on the lateral surface, connected by a rotation on the spot to correctly orient the cutter blade.

The planning of appropriate stiffness and trajectory is extremely important to successfully accomplish the task, especially in the latter case. In fact, wrong planning may lead to problems as the ones detailed in Fig.6: the cutter may tear off the plastic film if it rotates too late/is too rigid, and it may remain stuck against the top surface of the pallet if it rotates too early.

The problem of planning along the top and along the lateral surface is exactly dual. Consequently, let us analyze the case in which the cut is performed on the top surface of the pallet along the $\mathrm{X}$ direction (as in Fig.4). Note that the contact constraint in this case is represented by $L=\left[\begin{array}{lll}0 & 0 & 1\end{array}\right]$ with initial condition $z(0)=h$ and that $F_{e}=\left[\begin{array}{lll}F_{x} & F_{y} & 0\end{array}\right]^{T}$. Since the matrices in the dynamics (1) are all diagonal, the dynamic equations are decoupled. Hence, the problem can be split into three one dimensional planning problems.

\section{A. Planning in the Direction of the Contact}

In this section we address the problem of planning the stiffness and the trajectory in the direction perpendicular to the plane of the cut, namely $K_{z}$ and $z_{d}$. The problem can be formulated as:

$$
\begin{gathered}
\min _{K_{z}, z_{d}, F_{\perp}}\left\|F_{\perp}\right\| \\
\text { subject to: } \\
K_{z}\left(z-z_{d}\right)=F_{\perp} \\
\underline{K}_{z} \leq K_{z} \leq \bar{K}_{z} \\
0 \leq F_{\perp} \leq \bar{F} \perp \\
\underline{z} \leq z \leq \bar{z}
\end{gathered}
$$

where (2b) is given by (1) along $\mathrm{Z}$, considering that $z$ is constant due to the contact constraint, and that $z_{d}$ is chosen to be constant; equation (2c) is the interval of allowed values of $K_{z}$, with $\underline{K}_{z}>0 ; \bar{F}_{\perp}$ in (2d) is the maximum admissible value of the interaction force; equation (2e) is the interval

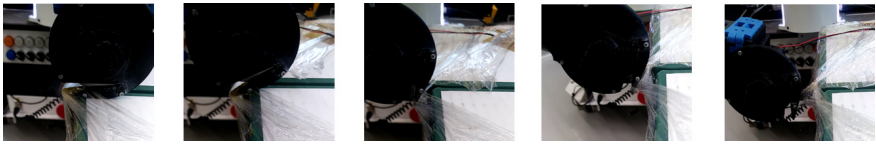

(a) The cutter rotates too late and it is not compliant enough.
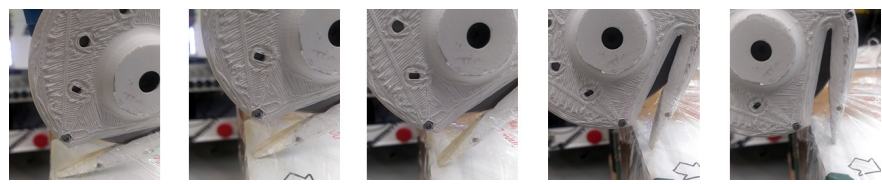

(b) The cutter rotates too early.

Fig. 6. Main problems caused by erroneous planning of the stiffness and of the trajectory.

inside which $z$ lies, and so, being $z=h$, it holds: $\underline{z}=\hat{h}-\Delta h$ and $\bar{z}=\hat{h}+\Delta h$. Constraint (2c) is necessary because too small values of impedance generate a poor trajectory tracking, due to non-perfect dynamic compensation (e.g. caused by internal friction) and external disturbances, while too high values may result in unstable behavior of the robot. Problem (2) is a robust optimization problem, being $z=h \in[\hat{h}-\Delta h, \hat{h}+\Delta h]$ a constant but uncertain parameter. The solution to the unconstrained problem would be trivially $F_{\perp}=0$. This would mean to have $z_{d}=z$, whose exact value we do not know, or to set $K_{z}=0$, which violates (2c).

On the other hand, for the constrained problem, given the definition of the cost function, we fix $K_{z}=\bar{K}_{z}$, so that only the desired trajectory has to be planned. In [16] the authors face a robust optimization problem aiming to minimize the interaction force between a linear system and a manipulated object; the interaction force is an external force for the system, that is a linear function of the state and of the control. The force, to be minimized by planning a desired trajectory (the impedance value being fixed), is affine in an uncertain parameter, which is the full state or part of it, for instance the object position. Our problem is included in the problems tackled in [16], where it is shown that the optimization problem can be split into two deterministic optimization ones, in which the uncertainty is at its bounds. Moreover, in [16] it is shown that the worst performing deterministic solution is the solution also to the robust optimization problem. Consequently, we solve the two problems where the uncertain parameter is at its bound. Substituting (2b) into the left hand side of (2d) leads to $K_{z}\left(z-z_{d}\right) \geq 0$, thus:

$$
z_{d} \leq z
$$

If we consider now the two deterministic problems where the uncertain parameter $z$ is at its lower and upper bounds, (3) becomes: $z_{d} \leq \underline{z}$ in one case, and $z_{d} \leq \bar{z}$ in the other. Consequently, since in order to minimize the cost function we desire the value of $z_{d}$ that is the closest to $\mathrm{z}$, the solutions of the two problems are:

$$
\left\{\begin{array}{l}
z_{d}=\underline{z} \\
K_{z}=\underline{K}_{z}
\end{array} \quad(4), \quad\left\{\begin{array}{l}
z_{d}=\bar{z} \\
K_{z}=\underline{K}_{z},
\end{array}\right.\right.
$$

Hence, for what has been said in [16], the solution of the deterministic problem that we pick is (4), which is the 


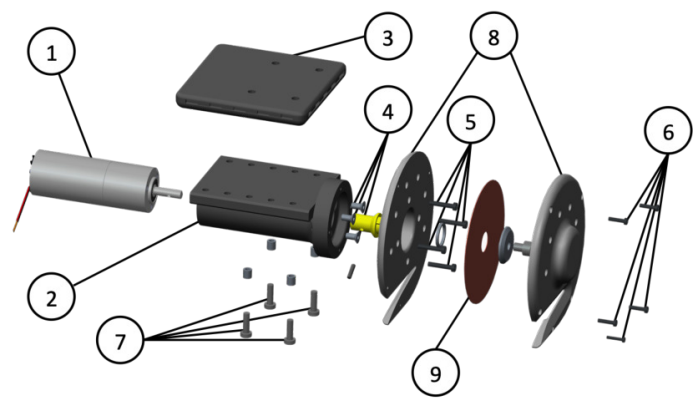

Fig. 7. Main components of the cutter end-effector
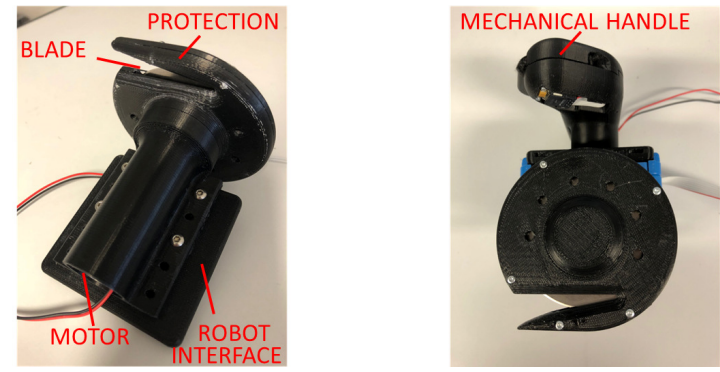

Fig. 8. On the left, a picture of the cutter prototype; on the right, a version provided with an handle for the manual use.

worst performing one w.r.t. the cost function. We conclude that the solution given by (4) is also solution of the robust optimization problem. Note that condition (4) assures the contact between the cutter and the pallet in the worst case scenario, namely when the height of the pallet is the smallest within the uncertainty bound.

Furthermore, we are able to define a feasibility condition, that can be used a priory to test if problem (2) admits a solution. Using (4), and $z=\bar{z}$, the right-hand side of constraint (2d) becomes:

$$
\underline{K}_{z}(\bar{z}-\underline{z}) \leq \bar{F}_{\perp},
$$

which relates the performance of the perception system (that determines the displacement between $\bar{z}$ and $\underline{z}$ ) and the maximum force that can be exerted on the items. Moreover, it links these aspects also with the performance of the control, which is responsible for the definition of the lower bound on $K_{z}$. In fact, the better the dynamic compensation is, the lower the impedance can be set without suffering from too large position errors.

\section{B. Planning in the Plane of the Cut}

In this section, we address the problem of planning the stiffness and the trajectory in the plane of the cut, namely $x_{d}(t)$ and $K_{x}$, and $y_{d}(t)$ and $K_{y}$. We start from the first two. We suppose to have a bounded external disturbance $F_{x}=F_{f}+F_{p}$, where $F_{f} \leq \mu F_{\perp}$ is the dynamic friction, being $\mu$ a constant coefficient, and $F_{p}$ is the force exerted by the plastic film on the cutter. Both the forces, whose nominal values $\hat{F}_{f}$ and $\hat{F}_{p}$ are assumed to be known, lie within certain known bounds. Our control objective is to achieve a bounded steady state error (bounded by $\epsilon \in \mathbb{R}^{+}$) under bounded external disturbances. $x_{d}(0)=X_{0}$ and $x_{d}(T)=X_{T}$ are the initial and final conditions for the cutter desired trajectory, and we choose $X_{T}=\hat{\ell}$, namely we end the trajectory at the estimated position of the pallet edge. More specifically, our objective is: $\lim _{t \rightarrow \infty}\left|x(t)-x_{d}(T)\right| \leq \epsilon$. The problem of planning $x_{d}(t)$ and $K_{x}$ could be solved in the simplest way by assigning $x_{d}(t)=X_{T}$ and by increasing the value of $K_{x}$ until the steady state error is less than the desired threshold, $\epsilon$. Consider the case in which the external forces are at their maximum value: $\bar{F}_{f}$ and $\bar{F}_{p}$, such that their sum gives $\bar{F}_{x}$. To ensure that $\lim _{t \rightarrow \infty}\left|x(T)-X_{T}\right| \leq \epsilon$, from the dynamics in steady state conditions, we have that: $K_{x} \geq \bar{F}_{x} / \epsilon$. Another possibility is for instance the one that assigns $x_{d}=X_{T}+x_{d}^{\prime}$, where $x_{d}^{\prime}=-\frac{\hat{F}_{x}}{K_{x}}$ is a feedforward term that zeroes the steady state error under nominal conditions (nominal values of $F_{f}$ and $F_{p}$ that sums up to $\hat{F}_{x}$ ). If the uncertain value of the external force is centred around a nominal value different from zero, this allows to pick a lower value of $K_{x}$. In fact, in the worst case, we have: $K_{x}\left(x(T)-X_{T}+\frac{\hat{F}_{x}}{K_{x}}\right)=\frac{\bar{F}_{x}}{K_{x}}$, leading to:

$$
K_{x} \geq \frac{\left|\bar{F}_{x}-\hat{F}_{x}\right|}{\epsilon} .
$$

We prefer this strategy, since a lower value of $K_{x}$ makes the robot softer, hence it helps in preventing disruptions of the plastic film in case the cutter goes beyond the pallet corner due to an overestimation of the corner position provided by the perception system (i.e. as in Fig. 6(a)).

Note that also $K_{x}$, as already discussed for $K_{z}$, must be kept within the interval $\left[\underline{K}_{x}, \bar{K}_{x}\right]$. Hence, we can define a feasibility condition: $\frac{\left|\bar{F}_{x}-\hat{F}_{x}\right|}{\epsilon} \leq \bar{K}_{x}$.

The problem of planning along $\mathrm{Y}$ is the result of the same exact problem described for the $\mathrm{X}$ direction, in which, however: $y_{d}(0)=y_{d}(T)=Y_{0}$ are the initial and final conditions, and $y_{d}$ is kept constant during the cut execution. Being the position of the cutter kept constant, the nominal values of the external force $F_{Y}$ can be considered zero. Thus, in this direction the simplest solution, namely $y_{d}=Y_{0}$ and $K_{y} \geq \bar{F}_{y} / \epsilon$ is a reasonable choice as explained above (being the nominal value of the external force is zero).

\section{CutTer Design}

Taking inspiration from the strategies exploited by human operators, we first tested existing tools, based on different working principles, to evaluate their performance and problems (see the Appendix for more details). The objects on which the tools have been tested are taken from the food industry, being one of the main end-users within the EUfunded project ILIAD a food company. The list of objects includes cardboard boxes of different sizes, plastic buckets, and metal cans. On the basis of these tests, we decided to adopt a concealed round actuated blade. The protection has been sized for two purposes: prevent the contact between the objects and the blade, facilitate the engagement between the cutter and the film. Furthermore, a protected blade is safer to be used in a human-robot shared environment. The size of the cutter is $140 \times 130 \times 40 \mathrm{~mm}$ and has been designed 

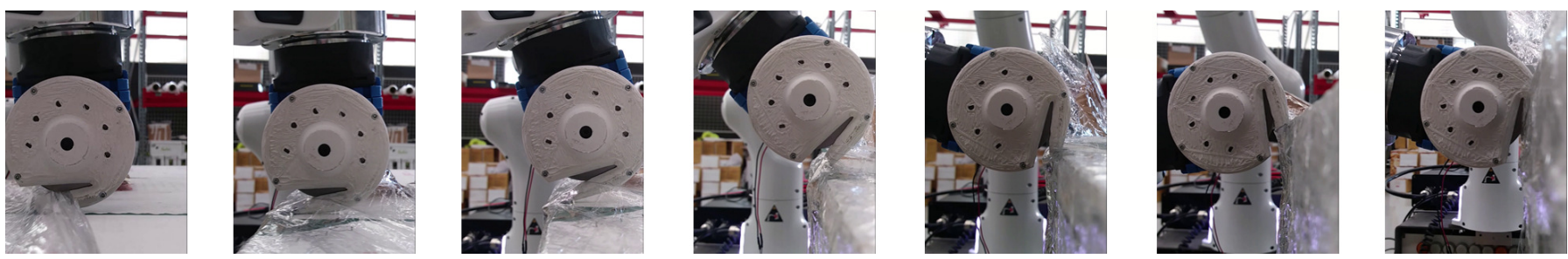

Fig. 9. Cutter Trajectory during an experiment in the incomplete top wrapping case.

in accordance to that of the best performing tools among the tested ones. The total mass is $0.35 \mathrm{~kg}$. The device is equipped with a custom electronic board, see [17] (details can be found on the NMMI website ${ }^{7}$ ).

Fig. 7 shows an exploded view of the 3-D model of the proposed cutter. The cutter is attached to the robot via the flange (3), that supports the housing (2) through the connection elements (7). The frame actuation unit (1), composed of an electric motor Maxon Motor DCX22S (12V), and provided with a planetary gearbox GPX22 14:1, is connected to the housing by (4) and moves the circular cutting element (9). The adopted blade is the one of the Bosch IXO tool, with a diameter of $60 \mathrm{~mm}$ a thickness of $0.6 \mathrm{~mm}$. It is protected by a frame (8) composed of two parts connected together by (6) and fixed to (2) through the connection elements (4 and 5). Figure 8 shows the cutter prototype (left), and a version of the cutter provided with a handle to execute the preliminary manual experimental tests (right), the results thereof are reported in the Appendix.

\section{EXPERIMENTAL VALIDATION}

\section{A. Experimental setup}

We used a Franka Emika Panda ${ }^{8}$ robot with our cutter as an end-effector. The robot is mounted on a mobile base, which uses a lidar for self-localization [18], [19] and an RGB-D camera for detecting the extents of the pallet. The perception system provides a segmented point cloud of the pallet (see Fig.9(a)). After filtering, a 2D bounding box approximation is derived for the visible faces of the pallet (e.g. see Fig.9(b)). To pick an initial cutting point, we currently assume to know the wrapping typology (i.e. Case 1 or Case 2 in Fig. 5) and the plastic film distribution. Fully automatic detection of the distribution of the film is the subject of ongoing work.

\section{B. Control}

In Sec. IV we derived the planning algorithm for impedance and trajectory assuming an impedance control law with a diagonal reshaped inertia matrix. This choice was adopted to simplify the algorithm derivation, allowing to solve three decoupled one-dimensional problems. In practical implementation a compliance controller (as the one described below) is often preferred because it does not require the feedback of the external wrench. This comes with the cost of a non-decoupled

\footnotetext{
${ }^{7}$ https://www.naturalmachinemotioninitiative.com/

${ }^{8} \mathrm{https}: / /$ www.franka.de/panda/
}

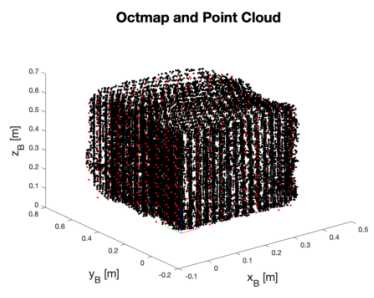

(a) The pallet Point Cloud

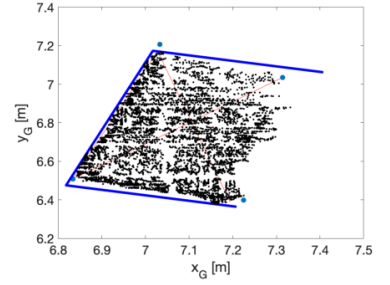

(b) 2D Boundary box around the top surface.
Fig. 10. Pallet information from the perception system

operational space dynamics. Indeed, the control control law implemented on out $\operatorname{robot}^{9}$ is (see Chapter 9 of [15]):

$$
u=g(q)+C(q, \dot{q}) \dot{q}+J(q)^{T} K\left(p_{d}-p\right)-J(q)^{T} D \dot{p},
$$

where $u \in \mathbb{R}^{n}$ is the control input torque, with $n=7$ is the number of joints; $q \in \mathbb{R}^{n}$ is the vector of generalized coordinates; $C(q, \dot{q})$ is the matrix of the Coriolis terms; $g(q)$ is the gravity vector; $J(q)$ is the Jacobian of the robot, $D$ and $K$ are desired stiffness and damping matrix as before; where the damping matrix $D$ is not a free parameter, but is set depending on the the stiffness $K$. Consequently, the closedloop operational space dynamics is:

$$
\begin{aligned}
& M(q) \ddot{p}+K\left(p-p_{d}\right)+(D+S(q, \dot{q})) \dot{p}=F_{e}+L^{T} F_{\perp}, \\
& L \dot{p}=0,
\end{aligned}
$$

where $\quad M(q)=\left(J(q) B(q)^{-1} J(q)^{T}\right)^{-1} \quad$ and $S(q, \dot{q})=-\left(J(q) B(q)^{-1} J(q)^{T}\right)^{-1} \dot{J}(q)$, being $B(q)$ the inertia matrix. The closed loop dynamics in (9) is not decoupled, since matrix $M(q)$ is not diagonal in general, nor is $S(q, \dot{q})$. However, despite this difference, the experimental results presented at the end of this section show that the quality of the proposed strategy is not jeopardized. This is probably due to the fact that during the trials the robot motion was slow enough to make the dynamic coupling terms negligible.

\section{Experimental Results}

To evaluate the intensity of the external forces and of the uncertainties, and to validate the proposed planning strategy, we report the data of one of the preliminary tests, in which a cut along $\mathrm{X}$ on the top face of the pallet has been performed. In Fig. 11 the estimated forces are shown, together with $p$,

\footnotetext{
${ }^{9}$ code available online at: https://github.com/frankaemika/libfranka/ blob/master/examples/cartesian_impedance_control.cpp
} 

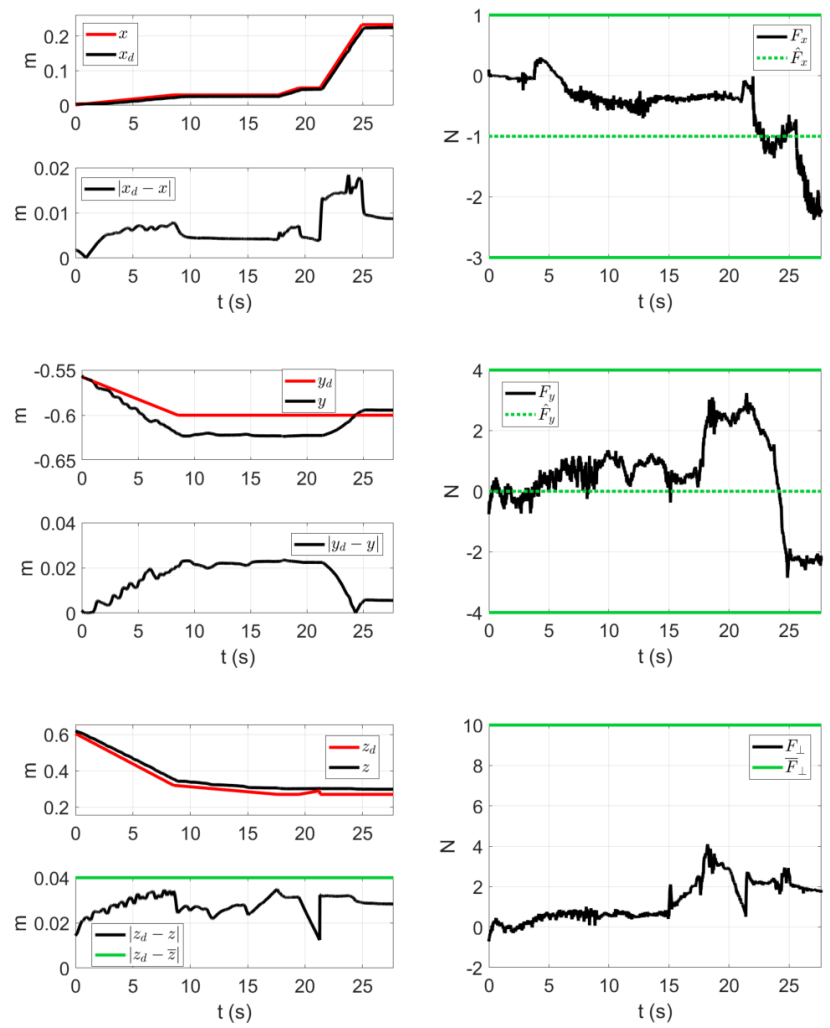

Fig. 11. The components of $p$ and $p_{d}$ are shown on the left, together with their difference, while the estimated forces are on the right. The task includes a first part during which the cutter reaches the point on the pallet from which the cut starts, and the cut itself begins from $t=25.47 \mathrm{~s}$.

$p_{d}$ and their difference. $\Delta h$ has been estimated equal to $0.02 \mathrm{~m}$, and $\bar{F}_{\perp}$ has been set to $10 \mathrm{~N}$, which is approximately the force exerted by an item characterized by a weight of $1 \mathrm{~kg}$ when it is put on top of another, and thus it can be regarded as a safe bound for $F_{\perp}$. Furthermore, we can say that $F_{x} \in\left[\hat{F}_{x}-2 \mathrm{~N}, \hat{F}_{x}+2 \mathrm{~N}\right]$, with $\hat{F}_{x}=-1 \mathrm{~N}$, and that $F_{y} \in\left[\hat{F}_{y}-4 \mathrm{~N}, \hat{F}_{y}+4 \mathrm{~N}\right]$, with $\hat{F}_{y}=0$. Note that, in the worst case, $\left|\bar{z}-z_{d}\right|=|\bar{z}-\underline{z}|=0.04 \mathrm{~m}$, hence the feasibility condition (6) is verified for $K_{z} \leq 250 \mathrm{~N} / \mathrm{m}$. We set $\epsilon= \pm 0.01 \mathrm{~m}$, both in $\mathrm{X}$ and $\mathrm{Y}$, since imposing a lower bound for the steady state error it would not be significant, given the uncertainty on the pallet size information. Consequently, we have that $K_{x} \geq 200 \mathrm{~N} / \mathrm{m}$ for (7), and, dually, $K_{y} \geq 400 \mathrm{~N} / \mathrm{m}$. For the experiments, $K_{x}=500 \mathrm{~N} / \mathrm{m}, K_{y}=400 \mathrm{~N} / \mathrm{m}$, and $K_{z}=200 \mathrm{~N} / \mathrm{m}$ have been chosen. The desired trajectory has been assigned as explained in the previous section. Note from Fig.11 that the proposed approach enables us to keep $F_{\perp}$ within acceptable values, and to keep the steady state error along $\mathrm{X}$ and $\mathrm{Y}$ below $\epsilon$.

Thanks to the described strategy, we are able to safely establish a contact with the object even though its position is not exactly known, and start the cut. Table I contains the outcome of the 26 performed tests, 13 for the Case 1 in Fig. 5, and 13 for the Case 2, with a duration of about 2.5 minutes each. The cuts in Case 1 are always successful. On the other hand, the experiments relative to Case 2 of Fig. 5 fail on three occasions, corresponding to underestimations of the position of
TABLE I

RESULTS OF THE LATERAL WRAPPING CASE (CASE1) AND INCOMPLETE TOP WRAPPING ONE (CASE2). THE CHECK MARK INDICATES A SUCCESSFUL CUT, AND THE CROSS A FAILURE.

\begin{tabular}{|c|c|c|c|c|}
\hline Test & Case $1=100 \%$ \\
\hline 001 & $\checkmark$ \\
\hline 002 & $\checkmark$ \\
\hline 003 & $\checkmark$ \\
\hline 004 & $\checkmark$ \\
\hline 005 & $\checkmark$ \\
\hline 006 & $\checkmark$ \\
\hline 007 & $\checkmark$ \\
\hline 001 & 1.69 & $\checkmark$ \\
\hline 002 & 0.17 & $\checkmark$ \\
\hline 003 & -1.29 & $\boldsymbol{\ell}$ \\
\hline 004 & -0.83 & $\checkmark$ \\
\hline 009 & $\checkmark$ \\
\hline 009 & $\checkmark$ \\
\hline 010 & $\checkmark$ \\
\hline 011 & $\checkmark$ \\
\hline 012 & $\checkmark$ \\
\hline 006 & -0.86 & $\checkmark$ \\
\hline 013 & $\checkmark$ & 2.13 & $\checkmark$ \\
\hline 008 & 0.29 & $\checkmark$ \\
\hline 009 & -1.46 & $\checkmark$ \\
\hline 010 & 1.3 & $\boldsymbol{X}$ \\
\hline 011 & -0.19 & $\checkmark$ \\
\hline 012 & 1.8 & $\checkmark$ \\
\hline 013 & -0.69 & $\boldsymbol{X}$ \\
\hline
\end{tabular}

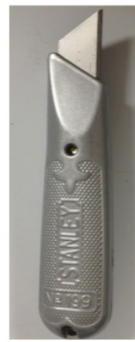

(a)

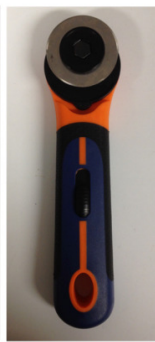

(b)

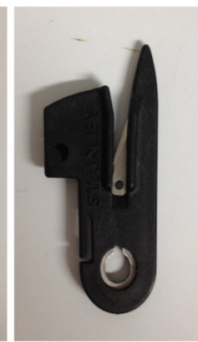

(c)

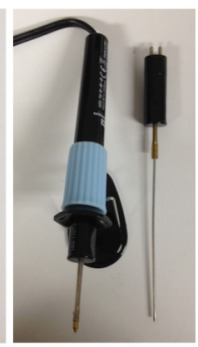

(d)
Fig. 12. Different kinds of unwrapping manual tools: a box cutter (a), a circular blade (b), a concealed blade (c), and a thermo-cut wire(d)

the pallet corner. The failure happens because the cutter rotates too early, remaining stuck against the pallet top surface, as in Fig. 6(b). This issue might be solved exploiting state of the art algorithms (e.g. [20]) to estimate the contact point on the cutter from contact wrench measurements. Such information may be used to update the planned trajectory. Instead, when the pallet corner position $\hat{\ell}$ is overestimated, the compliance conferred by $K_{x}$ results in the cutter being stopped by the plastic film in a position suitable for the turn.

\section{CONCLUSION AND FUTURE WORK}

This article provides a practically working mobile unwrapping system, which is an important step towards a completely integrated solution to efficient automatic unwrapping.

In the future, the presented setup will be integrated with automatic plastic film detection, and it will be made suitable for coping with generally structured pallets with irregular surfaces. For this purpose, the estimation of the contact point on the cutting end-effector, obtainable from wrench measurements, could be used as a basis for a reactive planner able to adapt to the irregular environment. With the insertion of such contact sensing, the system efficiency will hopefully be enhanced and, in view of practical applications, an evaluation of the system throughput will be carried out in real-world scenarios. Eventually, a range of additional application contexts will be considered, including the possibility of using tools based on a different cutting principle, e.g. the thermo-cut wire. Furthermore, with this respect, based on the end-user requirements, 


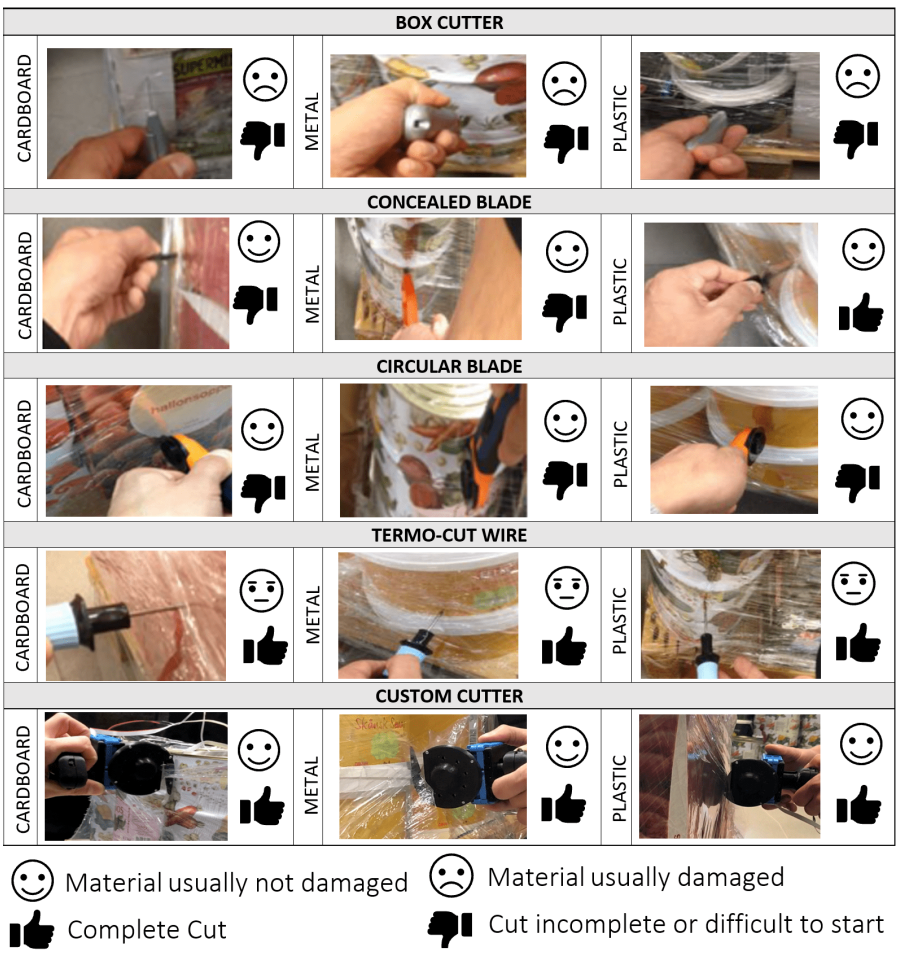

Fig. 13. Results of the manual tests with commercial blades and our cutter.

an additional prismatic joint located at the base of the arm will be added. This will enable vertical movements of the arm in order to enlarge the workspace and unwrap higher pallets. This might imply a lower precision in positioning the robot end-effector when the robot works at a considerable height, which we will address by increasing the uncertainty bounds of the pallet position. In the final version, the mobile base will be exploited in order to enhance the performance of the system and not only to have a relocatable unwrapping station.

\section{APPENDIX}

We describe here the results obtained testing different unwrapping tools manually. The most common tools have non-actuated blades to cut the wrapping films. The blade may be linear (Fig.12(a)) or circular (Fig.12(b)), and may have a protection in order not to damage the goods (Fig.12(c)). A different principle is based on a hot wire (Fig.12(d)) that may be used to weld the plastic film. In Fig.13, task pictures are shown, ordered by the material of the palletized items and the cutting tool that has been used, including the preliminary tests performed using our cutter manually. The main results are detailed in Fig. 13. We inferred that the box-cutter suffers from problems related to the task success (sometimes it is even difficult to start the cut), and it causes damage to the items. The concealed blade and the circular blade show a better behavior w.r.t. the damage to the items, but they usually do not complete the task successfully. The thermo-cut wire, instead, successfully accomplishes the task, though slightly damaging the items, but it is affected by different problems including the smell and the need for frequent cleaning. Taking into account an application of the system to the food industry scenario, we discarded the thermo-cut wire due to its drawbacks, which, however, might be tolerated in other application contexts. The design of our custom cutter is inspired by the tools in Fig. 12(b) and Fig. 12(c), with an actuated blade to boost the cut success.

\section{REFERENCES}

[1] W. Echelmeyer, A. Kirchheim, A. Lilienthal, H. Akbiyik, and M. Bonini, "Performance indicators for robotics systems in logistics applications," in IROS Workshop on Metrics and Methodologies for Autonomous Robot Teams in Logistics (MMARTLOG), 2011, p. 55.

[2] W. Echelmeyer, A. Kirchheim, E., and Wellbrock, "Robotics-logistics: Challenges for automation of logistic processes," in Automation and Logistics, 2008. ICAL 2008. IEEE International Conference on. IEEE, 2008, pp. 2099-2103.

[3] P. Günther and D. Arnold, "Intralogistik-eine starke branche stellt sich vor," Intralogistik-Potentiale, Perspektiven, Prognosen. Springer-Verlag, Berlin, Heidelberg, pp. 5-16, 2006.

[4] T. Wagner, C. Hausner, J. Elger, U. Lowen, and A. Luder, "Engineering processes for decentralized factory automation systems," in Factory Automation. InTech, 2010.

[5] F. Group, "World pallets," February 2014.

[6] I. Group, "Pallet market: Global industry trends, share, size, growth, opportunity and forecast 2018-2023," February 2018.

[7] P. M. Research, "Global pallets market global industry analysis 20122016 and forecast 2017-2025," June 2017.

[8] G. Garibotto, S. Masciangelo, M. Ilic, and P. Bassino, "Service robotics in logistic automation: Robolift: vision based autonomous navigation of a conventional fork-lift for pallet handling," in Advanced Robotics, 1997. ICRA'97. Proceedings., 8th International Conference on. IEEE, 1997, pp. 781-786.

[9] M. R. Walter, S. Karaman, E. Frazzoli, and S. Teller, "Closed-loop pallet manipulation in unstructured environments," in Intelligent Robots and Systems (IROS), 2010 IEEE/RSJ International Conference on. IEEE, 2010, pp. 5119-5126.

[10] R. Varga and S. Nedevschi, "Robust pallet detection for automated logistics operations." in VISIGRAPP (4: VISAPP), 2016, pp. 470-477.

[11] G. Baechler, I. Dokmanic, L. Baboulaz, and M. Vetterli, "Accurate recovery of a specularity from a few samples of the reflectance function," in 2016 IEEE International Conference on Acoustics, Speech and Signal Processing (ICASSP), Mar. 2016, pp. 1596-1600.

[12] M. Aittala, T. Weyrich, and J. Lehtinen, "Practical svbrdf capture in the frequency domain," ACM Trans. Graph., vol. 32, no. 4, pp. 110:1-110:12, Jul. 2013. [Online]. Available: http://doi.acm.org/10. $1145 / 2461912.2461978$

[13] N. Hogan, "Impedance control: An approach to manipulation," in American Control Conference, 1984. IEEE, 1984, pp. 304-313.

[14] A. Albu-Schaffer, C. Ott, U. Frese, and G. Hirzinger, "Cartesian impedance control of redundant robots: Recent results with the dlrlight-weight-arms," in IEEE International Conference on Robotics and Automation, vol. 3. IEEE; 1999, 2003, pp. 3704-3709.

[15] B. Siciliano, L. Sciavicco, L. Villani, and G. Oriolo, Robotics: modelling, planning and control. Springer Science \& Business M., 2010.

[16] G. M. Gasparri, F. Fabiani, M. Garabini, L. Pallottino, M. Catalano, G. Grioli, R. Persichini, and A. Bicchi, "Robust optimization of system compliance for physical interaction in uncertain scenarios," in 2016 IEEE-RAS 16th International Conference on Humanoid Robots (Humanoids). IEEE, 2016, pp. 911-918.

[17] C. D. Santina, C. Piazza, a. M. B. G. M. Gasparri, M. G. Catalano, G. Grioli, M. Garabini, and A. Bicchi, "The quest for natural machine motion: An open platform to fast-prototyping articulated soft robots," IEEE RAM, vol. 24, no. 1, pp. 48-56, 2017.

[18] T. Stoyanov, J. Saarinen, H. Andreasson, and A. J. Lilienthal, "Normal distributions transform occupancy map fusion: Simultaneous mapping and tracking in large scale dynamic e." 2013, pp. 4702-4708.

[19] J. Saarinen, H. Andreasson, T. Stoyanov, and A. J. Lilienthal, "Normal distributions transform monte-carlo localization (NDT-MCL)," 2013, pp. 382-389.

[20] A. Bicchi, J. K. Salisbury, D. L., and Brock, "Contact sensing from force measurements," The International Journal of Robotics Research, vol. 12 , no. 3, pp. 249-262, 1993. 